\title{
Puerarin protects endothelial progenitor cells from damage of angiotensin II via activation of ERK1/2-Nrf2 signaling pathway
}

\author{
CHEN FU $^{1}$, BAOXIN CHEN $^{1}$, XIANGLAN JIN ${ }^{1}$, XUEMEI LIU ${ }^{2}$, FENGLI WANG ${ }^{2}$, RONGJUAN GUO ${ }^{1}$, \\ ZHIGANG CHEN ${ }^{1}, \mathrm{HONG} \mathrm{ZHENG}^{2}, \mathrm{LE} \mathrm{WANG}^{1}$ and YUNLING ZHANG ${ }^{1}$ \\ ${ }^{1}$ Department of Neurology; ${ }^{2}$ Central Laboratory, Dongfang Hospital, \\ Beijing University of Chinese Medicine, Beijing 100078, P.R. China
}

Received June 26, 2017; Accepted August 31, 2017

DOI: $10.3892 / \mathrm{mmr} .2017 .8317$

\begin{abstract}
Endothelial progenitor cell (EPC) dysfunction is associated with the formation of carotid atherosclerosis. It has been demonstrated that angiotensin II (Ang II) may impair the function of EPCs and puerarin, a natural product, possesses cardiovascular protective effects against oxidative stress and inflammation. Therefore, the present study aimed to investigate the beneficial effects of puerarin in Ang II-induced EPC injury, and to elucidate the underlying mechanisms. Treatment with Ang II suppressed EPC proliferation and migration, increased the expression of the senescence marker $\beta$-galactosidase, and the adhesion molecules intracellular adhesion molecule-1 and vascular cell adhesion molecule-1. However, the above effects were markedly alleviated by treatment with puerarin in a dose-dependent manner $(1,10$ and $100 \mu \mathrm{M}$ ). In addition, Ang II significantly increased reactive oxygen species production and the levels of the inflammatory cytokine tumor necrosis factor- $\alpha$ and interleukin-6. Notably, these effects were reversed by puerarin. However, it was identified that the impaired EPC functions were due to inhibition of the phosphorylation of extracellular signal-regulated kinase 1 and 2 (ERK1/2) and the degradation of nuclear factor erythroid 2 like 2 (Nrf2), and treatment with puerarin activated the ERK1/2-Nrf2 signaling pathway. The results of the present study indicated that puerarin protected Ang II-induced
\end{abstract}

Correspondence to: Dr Le Wang or Dr Yunling Zhang, Department of Neurology, Dongfang Hospital, Beijing University of Chinese Medicine, 6 Zone One of Fangxingyuan, Fang Zhuang, Fengtai, Beijing 100078, P.R. China

E-mail: wangyspl155@163.com

E-mail: yunlingzzz@sina.com

Abbreviations: EPCs, endothelial progenitor cells; Ang II, angiotensin II; ROS, reactive oxygen species; $\beta$-gal, $\beta$-galactosidase; ICAM-1, intracellular adhesion molecule-1; VCAM-1, vascular cell adhesion molecule-1; TNF- $\alpha$, tumor necrosis factor- $\alpha$; ERK1/2, extracellular signal-regulated kinase 1/2; Nrf2, nuclear factor erythroid 2 like 2

Key words: EPCs, puerarin, Ang II, Nrf2
EPC dysfunction via activation of the ERK1/2-Nrf2 signaling pathway.

\section{Introduction}

Endothelial progenitor cells (EPCs) mobilized from bone marrow into the peripheral blood have been observed to serve an important role in endothelial repair and vascular regeneration by incorporating into the site of vessel injury, differentiating into endothelial cells, and releasing paracrine factors (1-3). Thereby, EPC depletion may lead to endothelial dysfunction. However, it has been demonstrated that the number of circulating EPCs in patients with atherosclerosis (AS) was decreased (4) and studies have reported that the number of EPCs may be associated with certain indicators of atherosclerosis, including intima-media thickness, in healthy adults $(5,6)$. Angiotensin II (Ang II), the main active effector of the renin-angiotensin system, serves an important role in the pathobiology of AS (7). A previous study demonstrated that Ang II is essential for EPC function, as Ang-II-induced oxidative stress causes senescence of EPCs and endothelial dysfunction (8). This, in turn, may accelerate the development of AS. A recent study demonstrated that natural extracts may restore the migration, adhesion and tube formation of EPCs, which are diminished by Ang II (9). This provided novel insights into the protection of EPCs from functional impairment.

Puerarin (4-7-dihydroxy-8-beta-D-glucosylisoflavone), one of the principal isoflavone glycosides extracted from the roots of Pueraria lobata, has been clinically used for the treatment of diseases, including hypertension, angina, myocardial infarction, arrhythmia, cerebral infarction and diabetes $(10,11)$. A number of studies have revealed that puerarin may protect the retina by inhibiting inflammation and neuronal damage induced by pro-inflammatory factors (12), may ameliorate endothelial dysfunction in isolated rat aortas (13), and may inhibit the endothelial inflammatory response (14). In addition, puerarin may function as a defender against Ang II-mediated oxidative stress-induced cell damage (15). The previous results described above suggested that puerarin may possess therapeutic potential for cardiovascular disorders, including AS. However, it remains unclear whether puerarin may serve an antioxidant role in Ang II-induced EPC damage. The present 
study, therefore, assessed the potential protective effects of puerarin on Ang II-mediated EPC injury, and investigated whether nuclear factor erythroid 2 like 2 (Nrf2) activation and extracellular signal-regulated kinase 1 and 2 (ERK1/2) phosphorylation may be involved.

\section{Materials and methods}

Identification of EPCs. EPCs were prepared as described previously (16). Peripheral blood mononuclear cells were obtained from the peripheral blood of ten healthy volunteers. Informed consent was provided by each participant and the above procedure was approved by the Ethics Committee of Dongfang Hospital (Beijing, China). Cells were isolated using Ficoll gradient centrifugation and cultured in endothelial growth medium-2 (EGM-2; Lonza Group, Ltd., Basel, Switzerland) supplemented with $10 \%$ fetal bovine serum (Gibco; Thermo Fisher Scientific, Inc., Waltham, MA, USA), in a humidified incubator with $5 \% \mathrm{CO}_{2}$ at $37^{\circ} \mathrm{C}$.

Following 7 days in culture, cells were incubated with Dil-acetylated low-density lipoprotein (AcLDL; $10 \mu \mathrm{g} / \mathrm{ml}$; Molecular Probes; Thermo Fisher Scientific, Inc.) at $37^{\circ} \mathrm{C}$ for $1 \mathrm{~h}$. Cells were fixed with $4 \%$ paraformaldehyde at room temperature for $10 \mathrm{~min}$ and subsequently incubated with fluorescein isothiocyanate-labeled lectin (UEA-1; $10 \mu \mathrm{g} / \mathrm{ml}$; Sigma-Aldrich; Merck KGaA, Darmstadt, Germany) for $1 \mathrm{~h}$. Following staining, the slides were observed under an inverted fluorescent microscope (Nikon Eclipse Ti-U; Nikon Corporation, Tokyo, Japan). Dual-stained cells (positive for Dil-ac-LDL and UEA-1) were identified as EPCs.

Treatment paradigm. For each experiment, cells were treated with human 1.0 $\mu \mathrm{M}$ Ang II (Sigma-Aldrich; Merck KGaA) for $24 \mathrm{~h}$ (17) with or without pre-treatment with various concentrations $(1,10$, and $100 \mu \mathrm{M})$ of puerarin $(99 \%$ purity as verified by high-performance liquid chromatography; Sigma-Aldrich; Merck KGaA) for $24 \mathrm{~h}$ (18). The molecular structure of puerarin is presented in Fig. 1. To identify whether the ERK1/2 signaling pathway was involved in the protective effect of puerarin, U0126 (10 $\mu \mathrm{M}$; Cell Signaling Technology, Inc., Danvers, MA, USA), a highly selective inhibitor of ERK1/2, was added to cell cultures 30 min prior to treatment with Ang II.

Cell proliferation assay. Cell proliferation was determined using the Cell Counting Kit-8 (CCK-8; Dojindo Molecular Technologies, Inc., Kumamoto, Japan) method. Cells were seeded into 96 -well plates at $1 \times 10^{4}$ cells/well and incubated in serum-free EGM-2 for $24 \mathrm{~h}$. Subsequently, media were removed, and cells were washed with PBS and stimulated with $1.0 \mu \mathrm{M}$ Ang II for $24 \mathrm{~h}$. To evaluate the effects of various concentrations of puerarin on the viability of EPCs, CCK-8 $(10 \mu \mathrm{l} / \mathrm{well})$ was added to the wells at the end of the experiment. Following incubation at $37^{\circ} \mathrm{C}$ for $2 \mathrm{~h}$, the absorbance of each well was determined using a microplate reader (Multiskan Spectrum; Thermo Fisher Scientific, Inc.) at $450 \mathrm{~nm}$. The degree of cell proliferation was expressed as the percentage absorbance of treated cells compared with control cells.

Cell migration assay. The effect of test substances on the migration of EPCs was determined using uncoated Transwell chambers. A density of $\sim 10^{6}$ cells was seeded into the upper chambers and medium containing $10 \%$ fetal bovine serum was used as a chemoattractant in the lower chambers. Following $24 \mathrm{~h}$ of incubation, the medium was removed, and the chambers were washed twice with PBS. The cells on the upper surfaces of the inserts were removed and those which had migrated to the lower surfaces were fixed with $4 \%$ paraformaldehyde for $10 \mathrm{~min}$ and stained with $0.1 \%$ crystal violet for $15 \mathrm{~min}$, both at room temperature. Microphotographs were obtained using a digital camera system (Olympus Corporation, Tokyo, Japan) at $\times 200$ magnification.

Measurement of reactive oxygen species (ROS) production and inflammation. For ROS detection, an Image-iT LIVE Green Reactive Oxygen Species Detection kit (Invitrogen; Thermo Fisher Scientific, Inc.) was used. EPCs (1x10 $/$ well) were incubated with EGM-2 containing $10 \mu \mathrm{M}$ 2,7-dichlorodihydrofluorescein diacetate for $30 \mathrm{~min}$ in 6-well plates and washed with PBS. The results were obtained and analyzed using a FACSCalibur ${ }^{\text {TM }}$ flow cytometer (BD Biosciences, San Jose, CA, USA).

Tumor necrosis factor (TNF)- $\alpha$ and interleukin (IL)- 6 concentrations in cell lysates were measured using TNF- $\alpha$ (cat. no. ab181421; Abcam, Cambridge, UK) and IL-6 (cat. no. ab46042; Abcam) enzyme-linked immunosorbent assay kits, according to the manufacturer's instructions. Experiments were repeated three times independently.

Western blot analysis. Treated cells were extracted using radioimmunoprecipitation lysis buffer (Biyuntian; Beyotime Institute of Biotechnology, Haimen, China) and protein concentrations were determined using the bicinchoninic acid method (Beyotime Institute of Biotechnology), according to the manufacturer's protocol. Protein $(30 \mu \mathrm{g})$ was separated by $10-12 \%$ SDS-PAGE and transferred to polyvinylidene fluoride membranes. Following blocking with $1 \%$ bovine serum albumin (MedChemExpress, Monmouth Junction, NJ, USA) at room temperature for $1 \mathrm{~h}$, the membranes were incubated with the following primary antibodies overnight at $4^{\circ} \mathrm{C}$ : Anti- $\beta$-galactosidase $(\beta$-gal; cat. no. ab168341; 1:500; Abcam), anti-intracellular adhesion molecule-1 (ICAM-1; cat. no. ab20; 1:1,000; Abcam) anti-vascular cell adhesion molecule-1 (VCAM-1; cat. no. ab134047; 1:5,000; Abcam), anti-ERK1/2 (cat no. 9102; 1:10,000; Cell Signaling Technology, Inc.), anti-phosphorylated (p-)ERK1/2 (cat no. 9101; 1:500; Cell Signaling Technology, Inc.), anti-Nrf2 (1:1,000; cat no. 12721, Cell Signaling Technology, Inc.) and anti-GAPDH (cat. no. ab8245; 1:1,000; Abcam). Subsequently, the membranes were incubated with anti-rabbit IgG horseradish peroxidase-conjugated secondary antibodies (cat. no. ab205718; 1:5,000; Abcam) for $2 \mathrm{~h}$ at room temperature. The immune complexes were detected using an Enhanced Chemiluminescence Plus kit (Biyuntian; Beyotime Institute of Biotechnology). Bands were then analyzed using the Quantity One 4.0 software (Bio-Rad Laboratories, Inc., Hercules, CA, USA). Each experiment was repeated three times independently.

Statistical analysis. All statistical analyses were performed using GraphPad Prism 6.5 (GraphPad Software, Inc., La Jolla, CA, USA). Data are presented as the mean \pm standard deviation. Comparisons among groups were performed using 


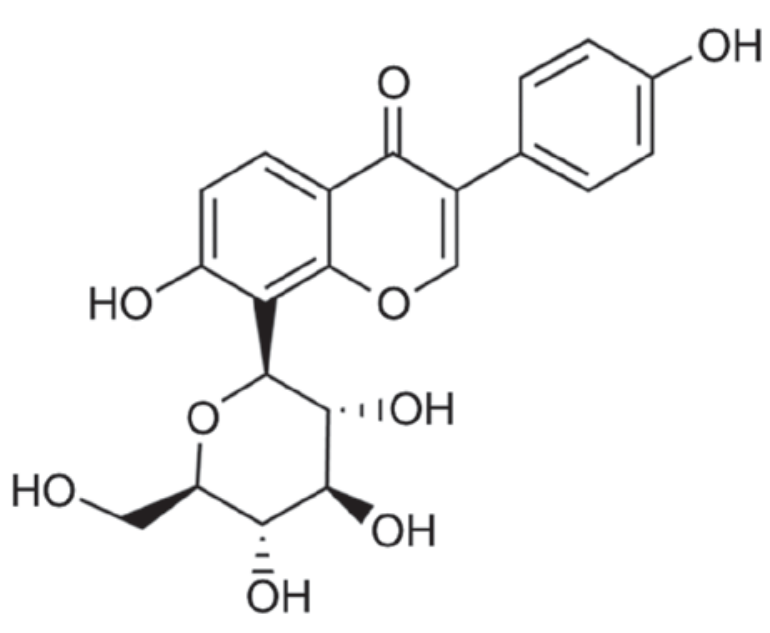

Figure 1. Molecular structure of puerarin.

one-way analysis of variance with post hoc Bonferroni or post hoc Dunnett tests. $\mathrm{P}<0.05$ was considered to indicate a statistically significant difference.

\section{Results}

Puerarin reverses the Ang II-induced inhibition of EPC proliferation and migration. EPCs were cultured from human peripheral blood mononuclear cells. Following 7 days in culture, isolated cells exhibited an endothelial cell-like morphology. As illustrated in Fig. 2, EPCs were characterized by double staining with Dil-AcLDL and UEA-1. Pre-incubation with 1.0 $\mu \mathrm{M}$ Ang II significantly suppressed EPC proliferation (Fig. 3A) and migration (Fig. 3B and C). However, puerarin reversed the inhibitory effect caused by $1.0 \mu \mathrm{M}$ Ang II, in a dose-dependent manner, on cell proliferation and migration (Fig. 3; $\mathrm{P}<0.05$ ).

Puerarin attenuates Ang II-induced EPCs senescence and adhesion. Compared with the control group, EPC senescence in the Ang II group was accelerated, as characterized by the increased level of $\beta$-gal (Fig. 4A and B). In addition, compared with the control group, the expression of ICAM-1 and VCAM-1, two adhesion molecules, was increased in the Ang II group (Fig. 4C and D). When EPCs were incubated with increasing concentrations of puerarin for $24 \mathrm{~h}$, the expression of $\beta$-gal was significantly decreased, in addition to the expression of ICAM-1 and VCAM-1 (Fig. 4; P<0.05).

Puerarin suppresses Ang II-induced oxidative stress and inflammation in EPCs. The results of the flow cytometry analysis presented in Fig. 5A demonstrated that Ang II significantly increased ROS production, compared with the control group, and its effects were attenuated by treatment with puerarin $(\mathrm{P}<0.05)$. In addition, the levels of TNF- $\alpha$ and IL-6 were significantly increased in the Ang II-treated EPCs compared with control EPCs. Similarly, puerarin reduced the expression of TNF- $\alpha$ and IL-6 in a dose-dependent manner (Fig. 5B and $\mathrm{C} ; \mathrm{P}<0.05$ ).

Puerarin alleviates Ang II-induced EPC damage by activating ERK1/2-Nrf2. In order to elucidate the mechanism underlying the above protective effects of puerarin on Ang II-induced
EPC damage, the present study investigated whether the ERK1/2-Nrf2 signaling pathway was involved in this regulation. EPCs were exposed to Ang II, puerarin or U0126, and western blotting was used to determine the protein levels of ERK1/2, p-ERK1/2 and Nrf2. Ang II induced a suppression of p-ERK1/2 and Nrf2 expression (Fig. 6). Notably, puerarin significantly increased the p-ERK1/2 expression level and reversed the suppression of $\mathrm{Nrf} 2$ protein expression mediated by Ang II. The effects of puerarin may be blocked by the ERK1/2 inhibitor U0126 (Fig. 6).

In addition, cell proliferation and migration following treatment with U0126 was decreased compared with treatment with Ang II and puerarin. However, there were no apparent alterations in the Ang II group compared with the Ang II + U0126 group or Ang II + puerarin + U0126 group (Fig. 7A). The effects of U0126 on cell senescence and adhesion (Fig. 7B and C) was consistent with the above. The levels of ROS production and inflammatory cytokines (TNF- $\alpha$ and IL-6) were also decreased when EPCs were exposed to U0126 (Fig. 7D-F). These results indicated that puerarin protected EPCs from Ang II-induced damage by activating ERK1/2 and Nrf2.

\section{Discussion}

The present study demonstrated that puerarin protected EPCs from Ang II-induced cell damage by decreasing ROS production and the expression of inflammatory cytokines. In addition, the results of the present study suggested that the protective effects of puerarin on EPC function may be dependent on the ERK1/2-Nrf2 signaling pathway.

In patients with carotid AS, it has been observed that the number of circulating EPCs is reduced and their function is impaired (19). EPCs may be a potential therapeutic target for vascular repair and regeneration, due to their strong capacity for proliferation and differentiation (20). However, studies have reported that EPCs may be damaged by Ang II and there is evidence that Ang II upregulates the levels of pro-inflammatory cytokines (including IL-6, monocyte chemoattractant protein-1 and VCAM-1) via the type 1 Ang II receptor, which may deteriorate the atherosclerotic inflammatory response $(21,22)$. Consistent with previous studies, the results of the present study suggested that cell proliferation and migration were decreased by Ang II, and it was demonstrated that $1.0 \mu \mathrm{M}$ Ang II stimulated ROS production to increase EPC senescence, and increased the expression of ICAM-1 and VCAM-1, two adhesion molecules of EPCs. Similarly, Han et al (23) demonstrated that Ang II caused EPC damage by testing proliferation, migration, adhesion, angiogenic capacity and tube formation in EPCs. Notably, the present study identified that puerarin was able to alleviate Ang II-mediated EPs injury.

There has been increasing interest in bioactive molecules that modulate cellular homeostasis and biological functioning. Puerarin, the principal isoflavone glycoside obtained from the root of Pueraria lobata (kudzu), has been observed to possess antioxidant (24), anti-hypercholesterolemic (25) and anti-hyperglycemic properties (26). In the present study, it was observed that puerarin had the ability to attenuate Ang II-induced EPC damage by decreasing ROS production. Similar to the results 

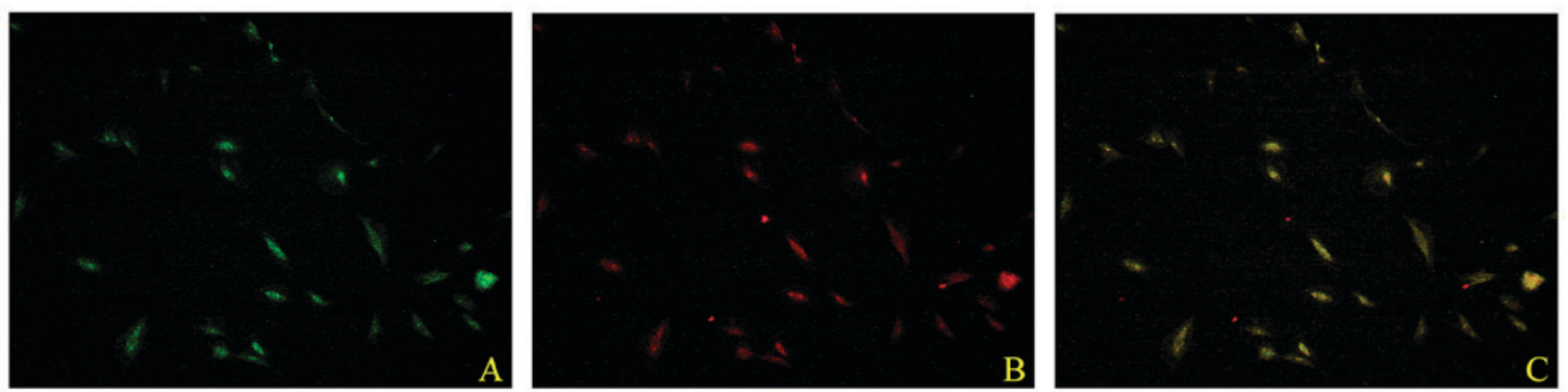

Figure 2. Identification of EPCs. Adherent cells exhibiting double-positive staining for UEA-1 (green; excitation wavelength, $488 \mathrm{~nm}$ ) and Dil-AcLDL (red; excitation wavelength, $549 \mathrm{~nm}$ ) were differentiated EPCs. (A) UEA-1; (B) Dil-acLDL; (C) merge. Magnification, x200. EPCs, endothelial progenitor cells.

A

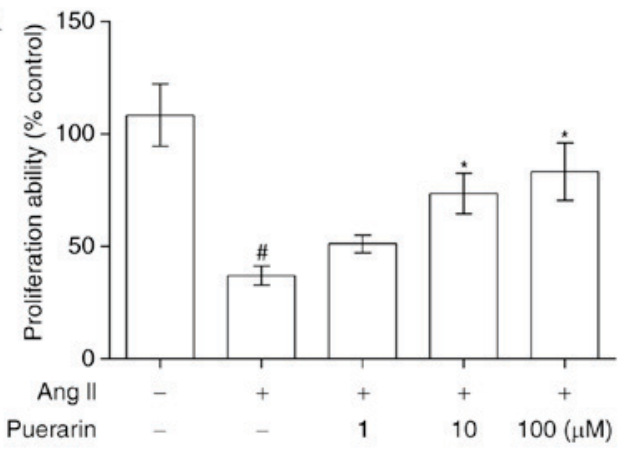

B

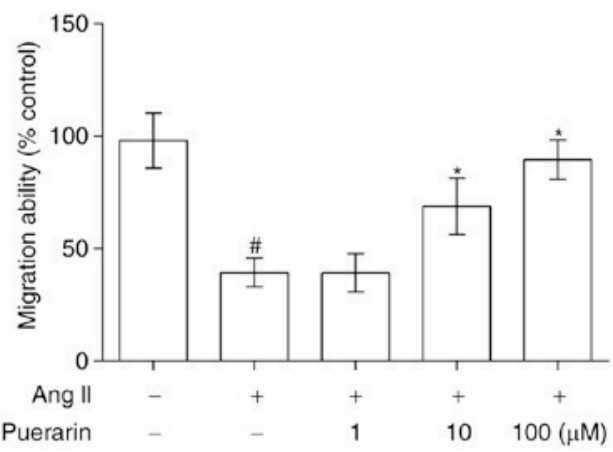

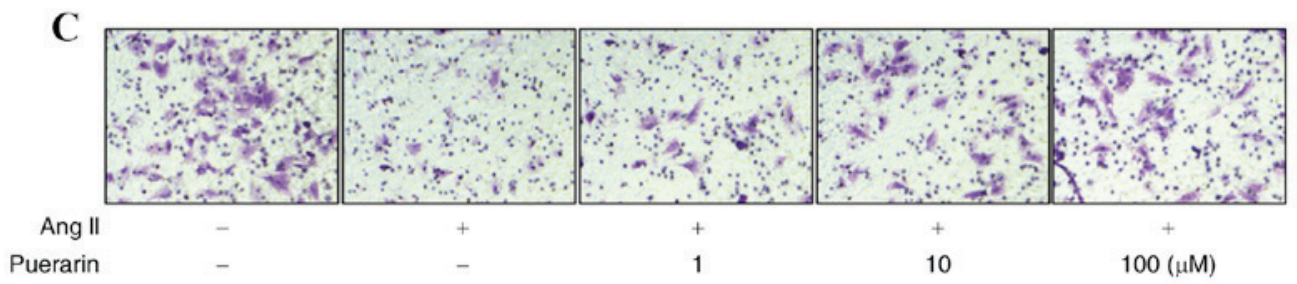

Figure 3. Effect of puerarin on the proliferative and migratory ability of EPCs induced by Ang II. (A) A Cell Counting Kit-8 assay demonstrated that Ang II inhibited EPC proliferation, and puerarin alleviated this inhibitory effect in a dose-dependent manner. (B) Puerarin eliminated the suppressive function of Ang II on EPCs migration in a dose-dependent manner. (C) Representative images of migratory cells. Magnification, $\mathrm{x} 200$. ${ }^{*} \mathrm{P}<0.05$ vs. control; " $\mathrm{P}<0.01 \mathrm{vs}$. Ang II. EPCs, endothelial progenitor cells; Ang II, angiotensin II.

$\mathbf{A}$

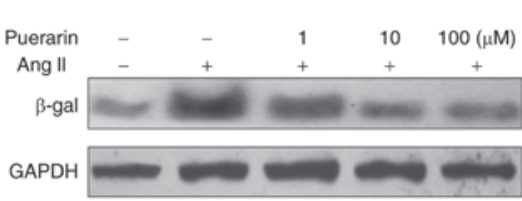

C

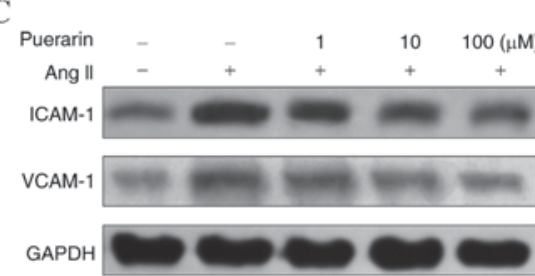

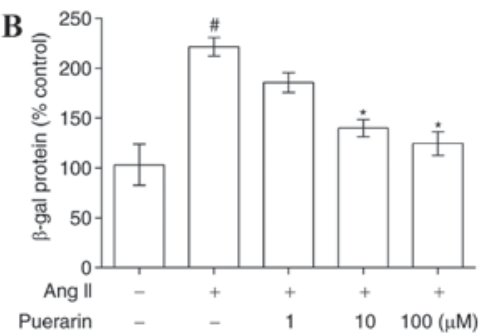

D

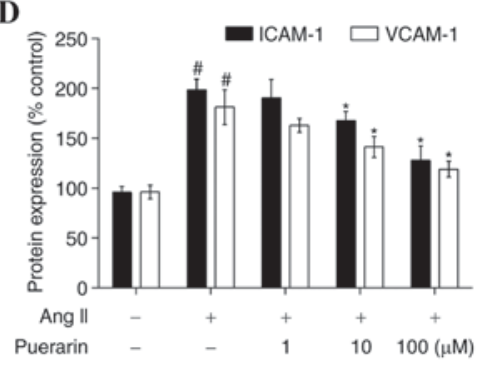

Figure 4. Effects of puerarin on the senescence and adhesion of Ang II-induced endothelial progenitor cells. The expression levels of $\beta$-gal were analyzed via (A) western blotting and (B) densitometric analysis. The expression of ICAM-1 and VCAM-1 were assessed via (C) western blot analysis and (D) densitometry. ${ }^{\#} \mathrm{P}<0.05$ vs. control; " $\mathrm{P}<0.01$ vs. Ang II. Ang II, angiotensin II; $\beta$-gal, $\beta$-galactosidase; ICAM-1, intracellular adhesion molecule-1; VCAM-1, vascular cell adhesion molecule-1. 

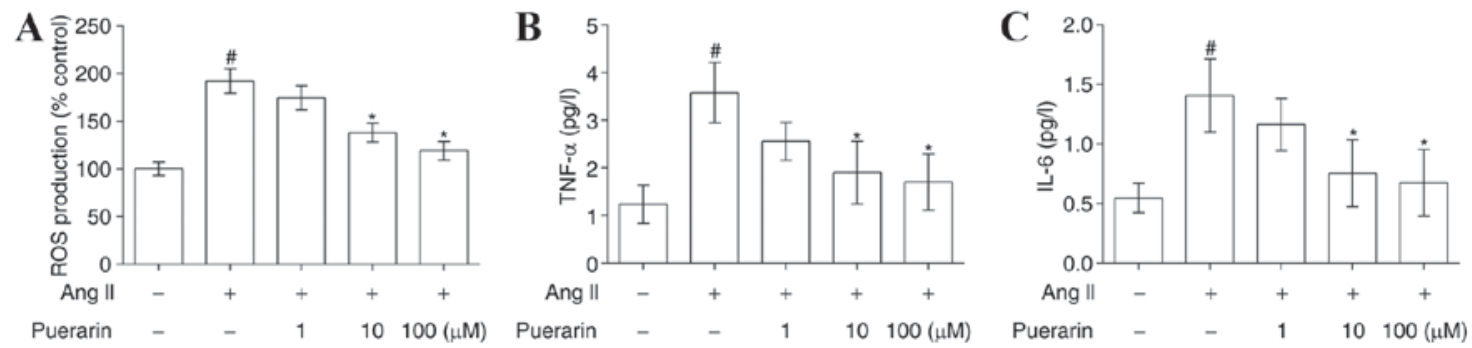

Figure 5. Effect of puerarin on EPC ROS production and inflammatory responses induced by Ang II. The ROS levels of EPCs were determined via 2,7-dichlorodihydrofluorescein diacetate staining and are expressed as a \% of the control group. ELISA analysis was used to quantify inflammatory factors. (A) Effect of puerarin on ROS levels detected by flow cytometry. (B) Effect of puerarin on TNF- $\alpha$ expression. (C) Effect of puerarin on IL- 6 expression. ${ }^{*} \mathrm{P}<0.05$ vs. control; " $\mathrm{P}<0.05$ vs. Ang II. EPCs, endothelial progenitor cells; ROS, reactive oxygen species; TNF- $\alpha$, tumor necrosis factor- $\alpha$; IL-6, interleukin-6; Ang II, angiotensin II.
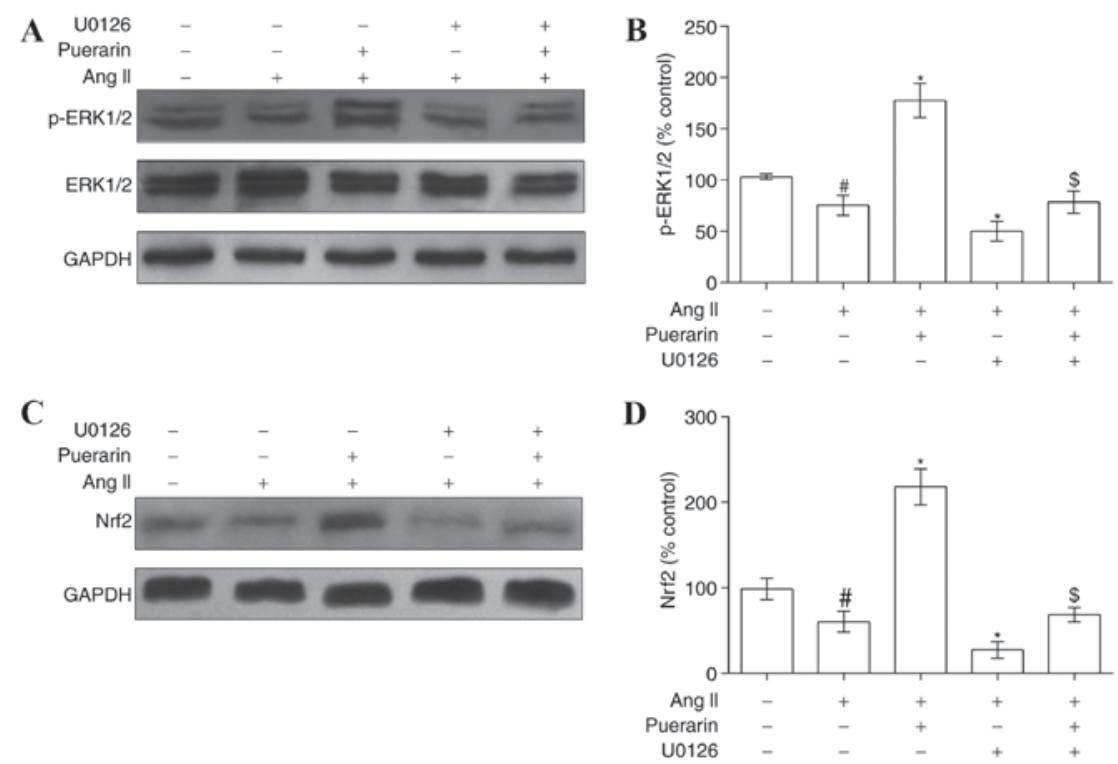

Figure 6. Effect of puerarin on the ERK1/2-Nrf2 pathway in endothelial progenitor cells induced by Ang II. U0126 is a selective inhibitor of ERK1/2. GAPDH was used to normalize protein loading. (A) The expression of p-ERK1/2 was examined using western blotting. (B) The ERK1/2 phosphorylation was calculated as the ratio of normalized arbitrary units of p-ERK1/2 over total ERK1/2. Nrf2 relative levels were detected by (C) western blot analysis and (D) densitometry. ${ }^{\#} \mathrm{P}<0.05$ vs. control; ${ }^{\mathrm{P}} \mathrm{P}<0.01$ vs. Ang II; ${ }^{\mathrm{S}} \mathrm{P}<0.05$ vs. Ang II + puerarin. ERK1/2, extracellular signal-regulated kinase $1 / 2$; p, phosphorylated; Ang II, angiotensin II; Nrf2, nuclear factor erythroid 2 like 2.
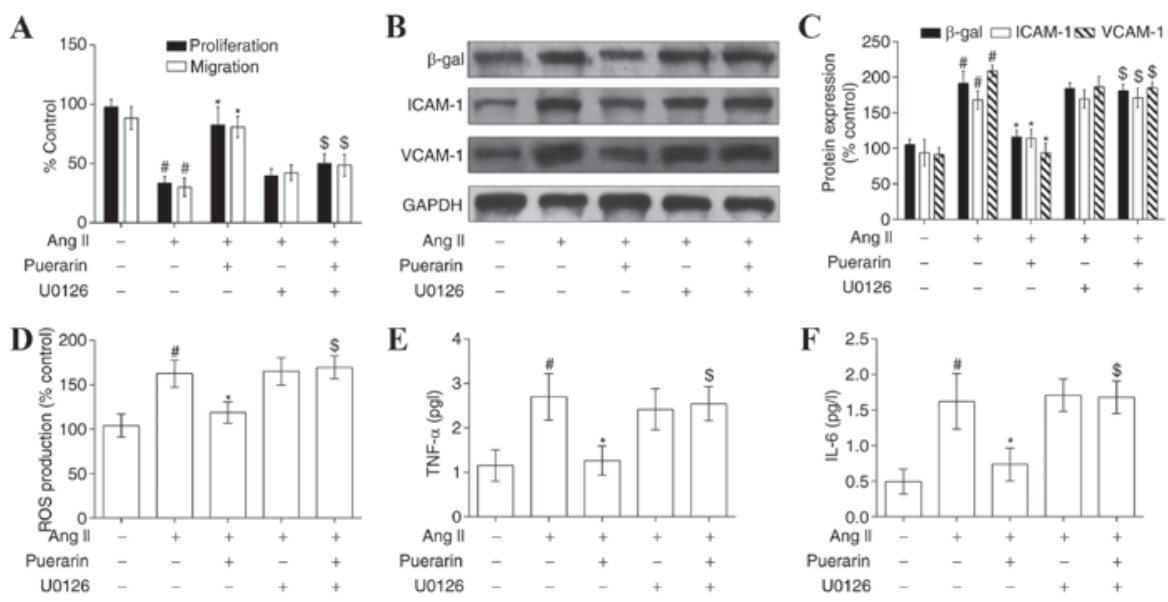

Figure 7. Puerarin attenuates Ang II-induced EPC dysfunction by activating the ERK1/2-nuclear factor erythroid 2 like 2 signaling pathway. (A) Cell proliferation and migration was measured in the different groups. The protein expression of $\beta$-gal, ICAM- 1 and VCAM- 1 was measured by (B) western blotting and (C) densitometry, following treatment with puerarin or U0126 (an inhibitor of ERK1/2) in Ang II-induced EPCs. (D) ROS levels were detected by flow cytometry in the different groups. (E) TNF- $\alpha$ and (F) IL-6 levels were detected by ELISA analysis in the different groups. ${ }^{~} \mathrm{P}<0.05$ vs. control; ${ }^{*} \mathrm{P}<0.01 \mathrm{vs}$. Ang II; ${ }^{5} \mathrm{P}<0.05$ vs. Ang II + puerarin. Ang II, angiotensin II; EPC, endothelial progenitor cell; ERK1/2, extracellular signal-regulated kinase; $\beta$-gal, $\beta$-galactosidase; ICAM-1, intracellular adhesion molecule-1; VCAM-1, vascular cell adhesion molecule-1; ROS, reactive oxygen species; TNF- $\alpha$, tumor necrosis factor- $\alpha$; IL-6, interleukin-6. 
of the present study, Lu et al (27) demonstrated that puerarin exerted its protective action via the reduction of NADPH oxidase-derived ROS overproduction and activation of the phosphatidylinositol 3-kinase (PI3K)/RAC- $\alpha$ serine/threonine protein kinase (Akt)/endothelial nitric oxide synthase (eNOS) pathways, in amyloid $\beta 40$ peptide-induced vessel impairment. In addition, emerging evidence has indicated the anti-inflammatory effects of puerarin in Ang II-induced endothelial dysfunction. Li et al (18) demonstrated that puerarin inhibited the expression of NADPH subunits and VCAM1, and increased the phosphorylation of eNOS at Ser 1177 in Ang II-infused rats. A study by Ji et al (28) demonstrated that puerarin inhibited the inflammatory response in atherosclerosis by suppressing the nuclear factor (NF) $-\kappa B$ signaling pathway. This previous study additionally demonstrated that puerarin induced the inhibition of adhesion molecules, including VCAM-1 and ICAM-1, which serve a critical role in AS $(29,30)$. The present study demonstrated that puerarin downregulated the Ang II-induced expression of ICAM-1 and VCAM-1 in EPCs. The mechanism of action of puerarin against Ang II-induced EPC injury was further investigated. As previously discussed, puerarin exerted antioxidant and cytoprotective effects via the activation of a number of signaling pathways, including the PI3K/Akt/eNOS (31), NF- $\mathrm{B}$ (28) and peroxisome proliferator-activated receptor pathways $(23,32)$. It was observed that, accompanied by the inhibition of inflammatory cytokines, puerarin activated the ERK1/2-Nrf2 signaling pathway in Ang II-induced EPCs. Subsequent chemical stressor analysis validated the hypothesis that puerarin exerted its cellular protective function by activating the ERK1/2-Nrf2 pathway. However, it remains to be completely understood whether puerarin may exert its function via other pathways and whether it affects EPC functioning in vivo.

In conclusion, the results of the present study demonstrated that puerarin activated the ERK1/2-Nrf2 signaling pathway, leading to cellular protection in EPCs exposed to Ang II.

\section{Acknowledgements}

The present study was supported by the Projects of Special Construction of Scientific Research of National Chinese Medicine Clinical Research Base (grant no. JDZX2015287) and the Basic Research Business Project of Beijing University of Chinese Medicine (grant no. 2016-JYB-JSMS-056).

\section{References}

1. Yin Y, Liu H, Wang F, Li L, Deng M, Huang L and Zhao X: Transplantation of cryopreserved human umbilical cord blood-derived endothelial progenitor cells induces recovery of carotid artery injury in nude rats. Stem Cell Res Ther 6: 37, 2015.

2. Zhu S, Malhotra A, Zhang L, Deng S, Zhang T, Freedman NJ, Storms R, Peppel K, Goldschmidt-Clermont PJ and Dong C: Human umbilical cord blood endothelial progenitor cells decrease vein graft neointimal hyperplasia in SCID mice. Atherosclerosis 212: 63-69, 2010.

3. Ke X, Shu XR, Wu F, Hu QS, Deng BQ, Wang JF and Nie RQ: Overexpression of the $\beta 2 \mathrm{AR}$ gene improves function and re-endothelialization capacity of EPCs after arterial injury in nude mice. Stem Cell Res Ther 7: 73, 2016.

4. Briasoulis A, Tousoulis D, Antoniades C, Papageorgiou N and Stefanadis C: The role of endothelial progenitor cells in vascular repair after arterial injury and atherosclerotic plaque development. Cardiovasc Ther 29: 125-139, 2011.
5. Fadini GP, Coracina A, Baesso I, Agostini C, Tiengo A, Avogaro A and de Kreutzenberg SV: Peripheral blood CD34+KDR+ endothelial progenitor cells are determinants of subclinical atherosclerosis in a middle-aged general population. Stroke 37: 2277-2282, 2006.

6. Lau KK, Chan YH, Yiu KH, Li SW, Tam S, Lau CP, Kwong YL and Tse HF: Burden of carotid atherosclerosis in patients with stroke: Relationships with circulating endothelial progenitor cells and hypertension. J Human Hypertens 21: 445-451, 2007.

7. Lin H, Pan S, Meng L, Zhou C, Jiang C, Ji Z, Chi J and Guo H: MicroRNA-384-mediated Herpud1 upregulation promotes angiotensin II-induced endothelial cell apoptosis. Biochem Biophys Res Commun 488: 453-460, 2017.

8. Calò LA, Facco M, Davis PA, Pagnin E, Maso LD, Puato M, Caielli P, Agostini C and Pessina AC: Endothelial progenitor cells relationships with clinical and biochemical factors in a human model of blunted angiotensin II signaling. Hypertens Res 34: 1017-1022, 2011.

9. Parzonko A, Czerwińska ME, Kiss AK and Naruszewicz M: Oleuropein and oleacein may restore biological functions of endothelial progenitor cells impaired by angiotensin II via activation of Nrf2/heme oxygenase-1 pathway. Phytomedicine 20: 1088-1094, 2013

10. Wong KH,Li GQ,Li KM, Razmovski-Naumovski V and Chan K: Kudzu root: Traditional uses and potential medicinal benefits in diabetes and cardiovascular diseases. J Ethnopharmacol 134: 584-607, 2011.

11. Prasain JK, Peng N, Rajbhandari R and Wyss JM: The Chinese Pueraria root extract (Pueraria lobata) ameliorates impaired glucose and lipid metabolism in obese mice. Phytomedicine 20: 17-23, 2012.

12. Teng Y, Cui H, Yang M, Song H, Zhang Q, Su Y and Zheng J: Protective effect of puerarin on diabetic retinopathy in rats. Mol Biol Rep 36: 1129-1133, 2009.

13. Meng XH, Ni C, Zhu L, Shen YL, Wang LL and Chen YY: Puerarin protects against high glucose-induced acute vascular dysfunction: Role of heme oxygenase-1 in rat thoracic aorta. Vascul Pharmacol 50: 110-115, 2009.

14. Hu W, Zhang Q, Yang X, Wang Y and Sun L: Puerarin inhibits adhesion molecule expression in tnf-alpha-stimulated human endothelial cells via modulation of the nuclear factor kappaB pathway. Pharmacology 85: 27-35, 2010.

15. Chen G, Pan SQ, Shen C, Pan SF, Zhang XM and He QY: Puerarin inhibits angiotensin II-induced cardiac hypertrophy via the redox-sensitive ERK1/2, p38 and NF-kappaB pathways. Acta Pharmacol Sin 35: 463-475, 2014.

16. Hill JM, Zalos G, Halcox JP, Schenke WH, Waclawiw MA, Quyyumi AA and Finkel T: Circulating endothelial progenitor cells, vascular function, and cardiovascular risk. N Engl J Med 348: 593-600, 2003.

17. Parzonko A, Oswit A, Bazylko A and Naruszewicz M: Anthocyans-rich Aronia melanocarpa extract possesses ability to protect endothelial progenitor cells against angiotensin II induced dysfunction. Phytomedicine 22: 1238-1246, 2015.

18. Li X, Lin Y, Zhou H, Li Y, Wang A, Wang H and Zhou MS: Puerarin protects against endothelial dysfunction and end-organ damage in Ang II-induced hypertension. Clin Exp Hypertens 39: 58-64, 2017.

19. Gong X, Shao L, Fu YM and Zou Y: Effects of olmesartan on endothelial progenitor cell mobilization and function in carotid atherosclerosis. Med Sci Monit 21: 1189-1193, 2015.

20. Yoder MC: Endothelial progenitor cell: A blood cell by many other names may serve similar functions. J Mol Med (Berl) 91: 285-295, 2013.

21. Bian F, Cui J, Zheng T and Jin S: Reactive oxygen species mediate angiotensin II-induced transcytosis of low-density lipoprotein across endothelial cells. Int J Mol Med 2017.

22. Li W, Li Z, Chen Y, Li S, Lv Y, Zhou W, Liao M, Zhu F, Zhou Z, Cheng X, et al: Autoantibodies targeting AT1 receptor from patients with acute coronary syndrome upregulate proinflammatory cytokines expression in endothelial cells involving NF- $\kappa \mathrm{B}$ pathway. J Immunol Res 2014: 342693, 2014.

23. Han T, Liu M and Yang S: DJ-1 alleviates angiotensin II-induced endothelial progenitor cell damage by activating the PPAR $\gamma / \mathrm{HO}-1$ pathway. J Cell Biochem 119: 392-400, 2018.

24. Xiong FL, Sun XH, Gan L, Yang XL and Xu HB: Puerarin protects rat pancreatic islets from damage by hydrogen peroxide. Eur J Pharmacol 529: 1-7, 2006.

25. Yan LP, Zhuang YL, Chan SW, Chen SL and Shi GG: Analysis of the mechanisms underlying the endothelium-dependent antivasoconstriction of puerarin in rat aorta. Naunyn Schmiedebergs Arch Pharmacol 379: 587-597, 2009. 
26. Hsu FL, Liu IM, Kuo DH, Chen WC, Su HC and Cheng JT: Antihyperglycemic effect of puerarin in streptozotocin-induced diabetic rats. J Nat Prod 66: 788-792, 2003.

27. Lu XL, Liu JX, Wu Q, Long SM, Zheng MY, Yao XL, Ren H, Wang YG, Su WW and Fai Cheung RT: Protective effects of puerarin against Aß40-induced vascular dysfunction in zebrafish and human endothelial cells. Eur J Pharmacol 732: 76-85, 2014.

28. Ji L, Du Q, Li Y and Hu W: Puerarin inhibits the inflammatory response in atherosclerosis via modulation of the NF- $\kappa \mathrm{B}$ pathway in a rabbit model. Pharmacol Rep 68: 1054-1059, 2016.

29. Chang CC, Chu CF, Wang CN, Wu HT, Bi KW, Pang JH and Huang ST: The anti-atherosclerotic effect of tanshinone IIA is associated with the inhibition of TNF- $\alpha$-induced VCAM-1, ICAMand CX3CL1 expression. Phytomedicine 21: 207-216, 2014.
30. Spigoni V, Picconi A, Cito M, Ridolfi V, Bonomini S, Casali C, Zavaroni I, Gnudi L, Metra M and Dei Cas A: Pioglitazone improves in vitro viability and function of endothelial progenitor cells from individuals with impaired glucose tolerance. Plos One 7: e48283, 2012.

31. Hwang YP, Kim HG, Hien TT, Jeong MH, Jeong TC and Jeong HG: Puerarin activates endothelial nitric oxide synthase through estrogen receptor-dependent PI3-kinase and calcium-dependent AMP-activated protein kinase. Toxicol Appl Pharmacol 257: 48-58, 2011.

32. Kang OH, Kim SB, Mun SH, Seo YS, Hwang HC, Lee YM, Lee HS, Kang DG and Kwon DY: Puerarin ameliorates hepatic steatosis by activating the PPARalpha and AMPK signaling pathways in hepatocytes. Int J Mol Med 35: 803-809, 2015. 